\title{
Twenty years of JHEP
}

\section{Daniele Amati and Loriano Bonora}

International School for Advanced Studies (SISSA), Via Bonomea 265, 34136 Trieste, Italy

E-mail: daniele.amati@sissa.it, loriano.bonora@sissa.it

On July 1st of twenty years ago we launched the first issue of JHEP. It was a real challenge to try the adventure of a scientific journal thought and managed by scientists, independent of private or institutional publishers. The idea was that physicists who were performing the work, writing the papers, and refereeing those of their peers, could also edit them in an electronic format. Thus, at a limited cost which could be handled by academic or scientific institutions, giving open access to the published information, and avoiding the heavy burden at that time placed on our libraries.

In order to avoid the failure that - as we well remember - was predicted to us by publishing companies and institutions, we needed the active support of the scientific community. A support in collaborating to the scrutiny of papers and scientific policies but, mainly, in sending to us their good papers. We contacted in that sense several of our most active and renowned colleagues, restricting of course to our high energy physics field. We received many enthusiastic responses as can be specifically seen from the papers that appear in the first issues of the journal, as well as the respected names listed in the advisory and in the editorial boards featuring in the opening page of the first issue of JHEP attached to this letter.

We were optimists, but perhaps not as much as to foresee the success of the journal, which, in his first 20 years, turned out to be one of the most (if not the most) prestigious one in our field. Success for which - as first project chairman and executive editor we must essentially thank the scientific community who supported JHEP by considering it "their journal", as was our intention from the start.

In this occasion we would like to recall the important contribution of Marco Fabbrichesi who designed the software on which the journal is based, and the technical personnel who implemented it and made it work. It is our pleasure to mention also the efficient editorial office, who liaised with intelligence and sympathy with authors, referees and editors. And, of course, the subsequent JHEP Directors (Hector Rubinstein and Marc Henneaux) who reinforced with devotion and success the JHEP scientific standard and its connection with the community.

Open Access. This article is distributed under the terms of the Creative Commons Attribution License (CC-BY 4.0), which permits any use, distribution and reproduction in any medium, provided the original author(s) and source are credited. 


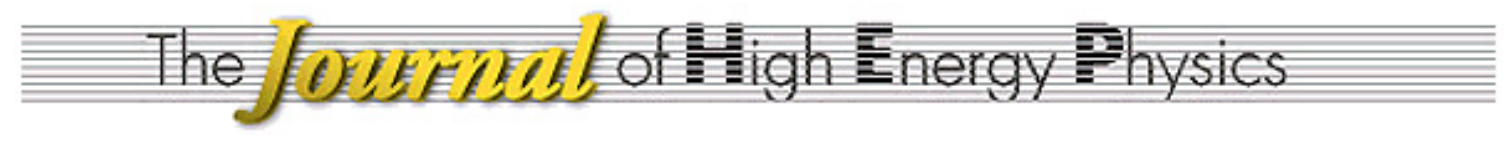

A refereed journal written, run and distributed by electronic means

Project chairman:

D. Amati (SISSA, Italy)

Advisory Board

J. Cardy (Oxford, UK)

S. Deser (Brandeis, USA)

R. Gatto (Genève, Switzerland)

D. Gross (ITP, USA)

L. Okun (ITEP, Russia)

G. Parisi (Roma, Italy)

G. 't Hooft (Utrecht, the Netherlands)

S. Weinberg (Austin, USA)

E. Witten (IAS, USA)

\section{Editorial Office}

L. Bonora (Executive Editor)

M. Candusso (Software Engineer)

S. Cerrato (Executive Editor)

M. Fabbrichesi (System Support)

M. Miculan (System Engineer)

F. Nesti (TeX Support)

\section{Editorial Board}

J. Ambjorn (NBI, Denmark)

P.S. Aspinwall (Rutgers, USA)

C. Bachas (Polytechnique, France)

J. Bagger (Johns Hopkins, USA)

R. Barbieri (Pisa, Italy)

J. Bijnens (Lund, Sweden)

R.H. Brandenberger (Brown, USA)

A. Cappelli (Firenze, Italy)

M.R. Douglas (Rutgers, USA)

B. Dubrovin (SISSA, Italy)

G.F. Giudice (CERN, Switzerland)

T. Eguchi (Tokio, Japan)

M. Kamionkowski (Columbia, USA)
L. Ibanez (Madrid, Spain)

J.M. Maldacena (Harvard, USA)

W.J. Marciano (BNL, USA)

S. Mukhi (Tata, India)

M. Neubert (CERN, Switzerland)

H. Ooguri (Berkeley, USA)

A. Pich (Valencia, Spain)

E. Rabinovici (Jerusalem, Israel)

V. Rittenberg (Bonn, Germany)

E. Roulet (Valencia, Spain)

A. Schwimmer (Weizmann, Israel)

M. Testa (Rome, Italy)

B. Webber (Cambridge, UK) 